S. Kwok, M. Dopita, and R. Sutherland, eds.

\title{
High resolution molecular hydrogen imaging of the Ring Nebula
}

\author{
A. K. Speck \& M. Meixner \\ Astronomy Dept., University of Ilinois at Urbana/Champaign, IL \\ 61801. \\ P. Knezek \& G. H. Jacoby \\ WIYN Observatory, Tucson, AZ 85726
}

The relative morphologies and structures of molecular and ionized gas emission from planetary nebulae ( $\mathrm{PNe}$ ) allow a better understanding of the nature and evolution of these objects. The classical paradigm for the structure of PNe is that of an ionized gas bubble bounded by neutral gas and molecules. However, it has been shown that molecular gas exists within ionized regions, leading to a re-evaluation of the classic structure. In the Helix Nebula (NGC 7293) dense condensations known as cometary knots are known to exist in the main ionized nebula. The molecules in these knots are shielded from the ionizing radiation and thus survive within the ionized zone. Another PN in which $\mathrm{H}_{2}$ emission is seen to originate from within the ionized nebula in NGC 6720 (the Ring Nebula).

We have presented new $\mathrm{H}_{2}$ images of the Ring nebula. The highest resolution image, which covers only the main, optically bright ring, was taken using the Near InfraRed IMager (NIRIM) at the WIYN telescope. This is the highest resolution image of molecular emission from the Ring nebula to date. A second image covers the entire nebula including the faint petal-structured inner halo and the fainter circular outer halo. This image, with a resolution of $1^{\prime \prime}$, was obtained using NIRIM at the Mount Laguna Observatory. The morphology of the molecular emission is compared to the morphology of the ionized emission from the Ring nebula as seen by the Hubble Space Telescope (He II, [O III] and $[\mathrm{N} \mathrm{II}])$ and the KPNO 4-m Mayall telescope $(\mathrm{H} \alpha)$.

The distribution of the molecular emission closely follows that of the ionized gas, displaying a bright central ring and the fainter petal structure. The resolution of the $\mathrm{H}_{2}$ observations in the main ring is sufficient to see the clumpy nature of the molecular gas. Comparing the high resolution $\mathrm{H}_{2}$ image with the Hubble Heritage image of the Ring, it is clear that the dark "globules" seen by the HST match the locations of clumpy $\mathrm{H}_{2}$ emission, suggesting that these "globules" are similar to the cometary knots seen in the Helix nebula. As with the Helix, the clumpy $\mathrm{H}_{2}$ emission from the main ring of the Ring nebula is entirely contained within the optically bright ionized nebula, implying that the molecular gas is shielded inside dense condensations. 